\title{
Reducing bacterial contamination of enteral feeds
}

\author{
C J Patchell, A Anderton, C Holden, A MacDonald, R H George, I W Booth
}

\begin{abstract}
Objective-It has previously been shown that microbial contamination of enteral feeds given to children in hospital and at home is common. This study therefore examined the effects of improvements in the enteral feeding protocol, coupled with an intensive staff training programme, on bacterial contamination.

Methods-The enteral feeding protocol was modified by: priming the feeding set on an alcohol treated metal tray, spraying the bottle opener and top with $70 \%$ alcohol, wearing non-sterile disposable gloves, and filling the feeding reservoir with feed for up to 24 hours' use rather than only four hours. Daily feeds samples were collected from 16 inpatients and home patients on enteral nutrition at the start and end of feeding. Seventy seven samples were cultured. Results were compared with previously published control data.

Results-Enteral feed contamination rates were reduced significantly from $62 \%$ to $6 \%$ of feeds given at home $(p<0.001)$, and from $45 \%$ to $4 \%$ of feeds given in hospital (p < 0.001).

Conclusions-This study highlights the importance of using an appropriate enteral feeding protocol, and of regular staff training in reducing contamination rates of enteral feeds to an acceptable level.

(Arch Dis Child 1998;78:166-168)
\end{abstract}

Keywords: enteral feeds; bacterial contamination

The Children's

Hospital, Birmingham

C J Patchell

C Holden

A MacDonald

R H George

Institute of Child

Health, University of

Birmingham

I W Booth

Department of

Bioscience and

Biotechnology,

University of

Strathclyde, Glasgow

A Anderton

Correspondence to: C J Patchell, Dietetic Department, Birmingham Children's Hospital NHS

Trust, Ladywood Middleway, Ladywood, Birmingham B16 8ET

Accepted 24 September 1997
Despite rigorous attempts to prevent contamination by the use of a single enteral feeding protocol across the hospital, and the training of staff and parents, unacceptably high levels of contamination were seen in all feeds. The protocol for the administration of enteral feeds specified that all feeds should be primed in the ward kitchen area, or for children on home treatment in the domestic kitchen, and that four hourly volumes of feed should be decanted into a giving set and the feed topped up at four hourly intervals.

Several possible risk factors were identified. These included the environment in which the feed was set up, inadequate hand washing techniques, ${ }^{6}$ poor attention to hygiene when handling the feed container and giving set, and repeated topping up of the feed at four hourly intervals. ${ }^{7-9}$ We therefore decided to adjust the enteral feeding protocol with the aim of minimising the risk of contamination, and to study the effects of the modification on the level of bacterial contamination of enteral feeds given in hospital and at home.

\section{Methods}

SUBJECTS

Feed samples were collected from 16 patients (mean age 2 years 10 months) receiving enteral feeds in hospital, and from five patients (mean age 4 years 7 months) being given home treatment. All patients were receiving Nutrison Paediatric Standard (Nutricia) as an enteral feed, prescribed as a supplement because of inadequate oral intake during the day, and were receiving enteral nutrition as a new treatment.

\section{FEEDING PROTOCOLS}

The feed was given overnight to all patients. The feeding system used was the Kangaroo System (Sherwood Medical) comprising a one litre nutrient container bag plus giving set.

The old and new enteral feeding protocols are summarised in table 1 . The feeding protocol was modified in the following way. For children in hospital, the feeding set was primed in the ward treatment area rather than the ward kitchen area, and feeds were set up on an alcohol treated metal trolley rather than a clean surface; for children receiving home enteral feeding, carers were advised to prime the feeding set on an alcohol treated metal tray. The bottle opener and feed bottle top were sprayed with $70 \%$ alcohol, and the feed bottle was wiped with alcohol. Non-sterile disposable gloves were worn. The reservoir was filled with up to 24 hours of feed rather than a four hour supply.

\section{STAFF TRAINING}

Revision of the feeding protocol was coupled with a period of intensive staff training, done jointly by one of the investigators (CJP) and a nutritional care sister. During these training 
Table 1 Old and new enteral feeding protocols

\begin{tabular}{|c|c|}
\hline Enteral feeding protocol (1994) & Revised enteral feeding protocol \\
\hline $\begin{array}{l}\text { (1) The feeding set should be primed } \\
\text { in a clean area, eg ward kitchen } \\
\text { (2) Ensure surfaces are clean } \\
\text { (3) Check feed expiry date } \\
\text { (4) Wipe enteral feeding pump with a } \\
\text { damp cloth } \\
\text { (5) Check alarms } \\
\text { (6) Remove feeding set from sterile } \\
\text { bag and check line } \\
\text { (7) Wipe the top of the feeding } \\
\text { container } \\
\text { (8) Wash hands using Ayliffe technique } \\
\text { (9) Fill the reservoir with } 4 \text { hours of } \\
\text { feed } \\
\text { (10) Prime the line ensuring air is } \\
\text { expelled } \\
\text { (11) Insert the feed line into the pump } \\
\text { ensuring the feeding set is properly } \\
\text { secured } \\
\text { (12) Check the position of the tube and } \\
\text { connect the giving set }\end{array}$ & $\begin{array}{l}\text { (1) The feeding set should be primed in the ward } \\
\text { treatment room } \\
\text { (2) Use a metal surface to set up feeds (treatment } \\
\text { trolley, or at home a metal tray, may be used). } \\
\text { Spray surfaces with } 70 \% \text { alcohol } \\
\text { (3) Check feed expiry date } \\
\text { (4) Wipe enteral feeding pump with a damp cloth } \\
\text { (5) Check alarms } \\
\text { (6) Remove feeding set from sterile and check line } \\
\text { (7) Wipe the top of the feeding container and spray } \\
\text { with } 70 \% \text { alcohol. Spray bottle opener with } \\
70 \% \text { alcohol } \\
\text { (8) Wash hands using Ayliffe technique. Put on } \\
\text { non-sterile disposable gloves } \\
\text { (9) Fill the reservoir with } 24 \text { hours of feed. Take } \\
\text { care to avoid touching the inner surface of the } \\
\text { reservoir } \\
\text { (10) Prime the line ensuring air is expelled } \\
\text { (11) Insert the feed line into the pump ensuring the } \\
\text { feeding set is properly secured } \\
\text { (12) Check the position of the tube and connect the } \\
\text { giving set }\end{array}$ \\
\hline
\end{tabular}

sessions, the nursing staff were made aware of the results of the original study and reasons for the modifications were discussed. The revised protocol was also used for training parents to feed their children enterally at home.

MICROBIOLOGICAL SAMPLING PROCEDURE Inpatients

Samples of enteral feed $(10 \mathrm{ml})$ were collected daily from:

(1) The lower end of the giving set before use, immediately after the feed had been decanted and the giving set assembled by nursing staff (start of administration).

(2) The nutrient container at the end of feeding. This feed sample was collected by a researcher spraying the giving set with $70 \%$ methylated spirit and cutting the portion of the giving set immediately below the nutrient container and above the drip chamber with alcohol treated scissors.

All samples were collected in sterile universal containers and were refrigerated at $<4^{\circ} \mathrm{C}$ immediately after collection. Microbiological analysis was carried out within 12 hours of collection.

Patients on home enteral nutrition

Weekly samples of the feed were collected in the home from:

(1) The nutrient container immediately before use (start of administration).

(2) The nutrient container at the end of feeding. This sample was collected by the same researcher using the method described above.
Carers were shown how to take samples at the start of administration and were asked to close the flow regulator and keep the entire giving set in a refrigerator at the end of administration, to be collected by the researcher on a home visit the same day.

MICROBIOLOGICAL ANALYSES

Viable counts were made on serial 10-fold dilutions of feed samples in $1 \%$ peptone water; $0.1 \mathrm{ml}$ of each dilution was spread over the surface of 5\% horse blood agar, MacConkey, and CLED agar (Oxoid). Plates were incubated aerobically at $37^{\circ} \mathrm{C}$ for 48 hours; counts were expressed as colony forming units (cfu)/ $\mathrm{ml}$. Isolates were identified by standard techniques.

\section{STATISTICS}

We calculated that we would need to examine 36 samples from each group of patients in order to detect a $50 \%$ reduction in feed contamination with a power of $95 \%$ and $\mathrm{p}<0.01$.

\section{Results}

SAMPLES FROM INPATIENTS

The hanging time for inpatient feeding systems varied between nine and 13 hours (median 11). Seventy seven samples were cultured. No contamination of enteral feed was detected at the start of administration, and only three out of 77 samples $(4 \%)$ were contaminated at the end of the administration period. Organisms isolated were Klebsiella aerogenes and coliforms at counts of $10^{3} \mathrm{cfu} / \mathrm{ml}$. This compares with $2 \%$ contamination of the sterile ready to use feed at the start of administration and $45 \%$ contamination at the end of the administration in the previous study - a highly significant improvement $(\mathrm{p}<0.001)$ in contamination rates (table 2$)$.

SAMPLES FROM CHILDREN ON HOME TREATMENT The hanging time for home feeding systems ranged from 10 to 14 hours (median 12 hours).

Thirty six samples were cultured. No contamination was detected at the start of administration and only two of 36 samples $(6 \%)$ were contaminated at the end of the administration period. The organism isolated was Streptococcus viridans at a count of $10^{6} \mathrm{cfu} /$ $\mathrm{ml}$. This compares with $28 \%$ of the ready to use feed being contaminated at the start of administration and $62 \%$ being contaminated at the end of administration in the previous studyagain a highly significant improvement in contamination rates $(\mathrm{p}<0.001)$ (table 2$)$.

Table 2 Contamination data; results are number (\%) of feeds contaminated

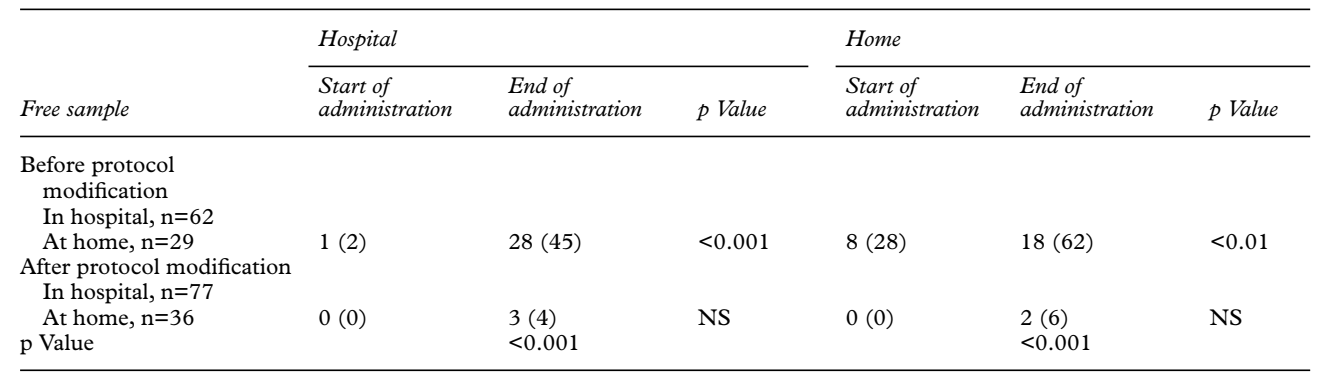




\section{Discussion}

The original study showed that high levels of contamination were seen in a sterile ready to use enteral feed when used both in hospital and at home, despite rigorous attempts to minimise contamination by the use of an enteral feeding protocol and regular staff training. Modification of the protocol for the second study and more intensive training led to a significant improvement and virtually eliminated contamination. The protocol was modified in various respects and it is not possible to identify which change was the most significant, or whether indeed the improvement resulted from the interaction of several factors.

The practice of topping up the feed every four hours in the original study was introduced because the feeds used are often modular and are not sterile. The theoretical advantage was that contaminated feeds should remain at room temperature for a minimum time and the bulk of the day's feed should be kept in a refrigerator until use. This practice, however, is now known to increase the risk of contamination significantly, since the giving set may be handled up to six times in a 24 hour period. $^{7}$ The change made to the protocol whereby all the day's feed requirement is decanted into the giving set is likely to be an important factor in the reduced contamination seen. We have now adopted this practice for all sterile ready to use feeds. It is, however, often necessary to modify enteral feeds or to use modular feeds for children with specialist nutritional requirements, and these feeds are prepared in a special feed unit. Such feeds are terminally heat treated by pasteurisation at $67^{\circ} \mathrm{C}$ for three minutes, but are not guaranteed to be sterile. For modular feeds it is therefore still necessary to decant four hourly quantities of feed into the giving set to minimise the time the feed is left at room temperature and thus reduce bacterial growth. The remaining feed is kept refrigerated until required.

Previous studies have shown that hands are a major source of contamination, because of poor hand washing techniques. ${ }^{8}$ Observation of hand washing at ward level and by parents in the home showed that techniques were often inadequate, or that hand washing was not done at all before setting up the enteral feeding system. This is in contrast to the practice observed when setting up intravenous feeding, when scrupulous hand washing techniques are used. This may be a reflection of the lower importance previously placed on the hygiene of enteral feeding systems by ward staff and parents.

The use of non-sterile disposable gloves is another important factor in improving hygiene, by providing an additional barrier to contamination. Anderton and Aidoo showed that contamination of enteral feeding systems could be eliminated in a laboratory situation by the use of non-sterile disposable gloves when handling the feed and feeding equipment. ${ }^{10}$

The bottle opener and feed bottle are other known sources of contamination, and studies have shown that disinfection of the bottle opener and feed container can eliminate feed contamination. ${ }^{7}$ This practice was therefore introduced into the revised protocol. Finally, increased staff awareness of the importance of hygiene in the setting up of enteral feeding systems may also be a factor responsible for the reduced contamination seen. The training sessions discussed the unacceptable levels of contamination isolated in the original study. Most members of ward staff were concerned by these results and considered them to be unacceptable.

Our results compare favourably with those of Puntis et al in a study examining intravascular catheter sepsis rates. Sepsis rates were reduced from $45 \%$ to $8 \%$ following protocol amendments and staff education. ${ }^{11}$

Contamination of enteral feeds is therefore probably multifactorial in origin and related to the number of manipulations of the feed and feeding system. In this study we have shown that bacterial contamination can be eliminated by modification of the enteral feeding protocol, which emphasises the key role that staff education plays in controlling contamination rates. Following this study, we now recommend that staff training should be an ongoing and continual process. Ward protocols are reviewed annually by the professional development unit, a body of senior nurses responsible for policies and procedures, and changes are disseminated to the ward staff by ward managers.

1 Patchell CJ, Anderton A, MacDonald A, et al. Bacterial contamination of enteral feeds. Arch Dis Child 1994;70:327-30. 2 Casewell MW, Cooper JE, Webster M. Enteral feeds contaminated with Enterobacter cloacae as a cause of septicaemia. BMF 1981;282:973.

3 Anderton A. Microbiological aspects of the preparation and administration of nasogastric and nasoenteric tube feeds in hospitals-a review. Hum Nutr: Appl Nutr 1983;37a:42640.

4 Levny J, Van Laethem Y, Verhaegan G. Contaminated enteral nutrition solutions as a cause of nosocomial blood stream infections. A study using plasmid finger printing. fournal of Parenteral and Enteral Nutrition 1981;5:76-9.

5 Thurn J, Crossley K, Gedts A, et al. Enteral hyper alimentation as a source of nosocomial infection. F Hosp Infect 1990; $15 \cdot 203-7$.

6 Ayliffe GAJ, Babb JR, Quoraishi AH. A test for hygienic hand disinfection. $\mathcal{F}$ Clin Pathol 1978;31:923.

7 Anderton A, Aidoo KE. Decanting - a source of contamination of enteral feeds. Clin Nutr 1990;9:157-62.

8 Anderton A, Aidoo KE. The effect of handling procedures on microbial contamination of enteral feeds. F Hosp Infect 1988;11: 364-72.

9 Anderton A, Howard JP, Scott EW. Microbiological control in enteral feeding. A guidance document. The Parenteral and Enteral Nutrition Group. London: The British Dietetic Association, 1986.

10 Anderton A, Aidoo KE. The effect of handling procedures on microbial contamination of enteral feeds - a comparison of the use of sterile vs non sterile gloves. F Hosp Infect 1991;17:297-301.

11 Puntis JWL, Holden CE, Smallman S, et al. Staff training: a key factor in reducing intravascular catheter sepsis. Arch Dis Child 1990;65:335-7. 